\title{
Immunadsorption und therapeutischer Plasmaaustausch
}

\author{
Welche Indikationen gibt es in Nephrologie und Neurologie?
}

\author{
Markus Tölle \\ CharitéCentrum13 - Schwerpunkt Nephrologie und \\ Nierentransplantationszentrum, \\ Campus Benjamin Franklin, Charité - Universitätsmedizin \\ Berlin
}

Bibliografie

DOI 10.1055/a-1011-5257

\begin{abstract}
ZUSAMMENFASSUNG
Die zur Verfügung stehenden Aphereseformen sind wirksame und komplementäre Therapieoptionen u. a. für die Behandlung unterschiedlicher Autoimmunerkrankungen. Neben dem therapeutischen Plasmaaustausch bedeutet die Immunadsorption einen wichtigen Fortschritt unserer Behandlungsmöglichkeiten. In vielen Bereichen der Medizin, insbesondere in der Nephrologie und der Neurologie, konnten autoimmunologische Mechanismen als Auslöser zahlreicher Erkrankungen identifiziert werden. Bei vielen dieser Krankheitsbilder stellt die therapeutische Apherese eine wertvolle Therapieoption dar, insbesondere bei ausgeprägter klinischer Symptomatik oder bei therapieresistenten Verläufen. Im Rahmen dieses Artikels wird auf den Einsatz der therapeutischen Apherese bei den wichtigsten nephrologischen und neurologischen Erkrankungen eingegangen.
\end{abstract}

\section{Einführung}

Die Immunadsorption (IA) und der therapeutische Plasmaaustausch (TPA) gehören zur Gruppe der therapeutischen Apheresen (TA). Als Apherese bezeichnet man Behandlungsverfahren, deren Therapieeffekt auf der extrakorporalen Elimination pathogener Proteine, proteingebundener Substanzen oder pathogener Zellen des Blutes beruht. Der Begriff stammt aus dem Griechischen und bedeutet „einen Teil aus einem Ganzen entfernen“.

Die zu diesem Zweck notwendige Auftrennung des Blutes in Plasma und seine zellulären Bestandteile erfolgt entweder mithilfe eines Membranplasmaseparators oder einer Hämozentrifuge [1]. Der geringere technische Aufwand und die Separation eines thrombozytenfreien Plasmas ist ein Vorteil der Membranplasmaseparation, die Bildung einer Sekundärmembran im Plasmafilter, die die Filtrationsleistung zeitlich begrenzt, ein Nachteil. Dagegen kann unter Nutzung einer Hämozentrifuge bei adäquater Antikoagulation zeitlich eine nahezu unbegrenzte Plasmamenge gewonnen werden, wobei sich die geringe Kontamination mit Thrombozyten negativ auf die Sekundärtrennung auswirken kann. Mit beiden Verfahren ist die Nutzung peripherer Venen prinzipiell möglich. Bei inadäquaten Blutflüssen ist die Anlage eines temporären Dialysekatheters notwendig. Bei langfristig notwendigen Behandlungen ist u. U. über eine Shuntanlage zu diskutieren.

Im Rahmen einer unselektiven Apherese wird das abgetrennte Plasma verworfen und i.d. R. entweder gegen Fremdplasma (PS: Plasmaseparation) oder gegen eine Hu- manalbuminlösung (PP: Plasmapherese) ersetzt. Im Rahmen einer selektiven Apherese werden dem abgetrennten Plasma durch Nutzung von Adsorption, Präzipitation oder Filtration gezielt Plasmabestandteile entzogen und dem Patienten das aufgereinigte Plasma wieder zugeführt. Auf eine Substitutionslösung kann hierbei verzichtet werden. Durch die unterschiedlichen Aphereseverfahren ist es zum einen möglich geworden, Pathogene, Komplementfaktoren, Zytokine, Chemokine, Fibrinogen, Antikörper (AK) und AK-Komplexe aus dem Plasma zu eliminieren [2-4] und zum anderen bedeutsame Plasmaproteine, wie ADAM-TS13, in großen Mengen zu substituieren [5]. Durch adsorptive Mechanismen kann sich darüber hinaus eine Änderung der immunologischen Funktion ergeben. So zeigen sich Änderungen der CD4/CD8-Ratio, des TH1/TH2-Verhältnisses oder der Funktion von regulatorischen T-Zellen.

Plasmakomponenten zeigen ein typisches Verteilungsvolumen, sodass vergleichbar zur Dialyse die Effektivität der Behandlung im zeitlichen Verlauf der TA aufgrund einer zeitverzögerten intravaskulären Wiederauffüllung nachlässt. So weisen bspw. Immunglobuline (Ig) eine unterschiedlich lange Halbwertzeit auf und verteilen sich in einem unterschiedlich großen Verhältnis im intra- und extravaskulären Raum ( $\vee$ Tab. 1). Das Wiederauffüllen des intravaskulären Raums erfolgt durch die lymphatische Drainage und die endogene Synthese.

Die Dynamik der Veränderung einer Plasmakonzentration soll an einer Modellsubstanz, einem Immunglobulin G 
- Tab. 1 Übersicht über die physiologischen Eigenschaften unterschiedlicher Plasmaproteine.

\begin{tabular}{|c|c|c|c|c|c|}
\hline Protein & $\begin{array}{l}\text { Konzentration } \\
\text { (mg/ml) }\end{array}$ & $\begin{array}{l}\text { Molekulargewicht } \\
\text { (kDa) }\end{array}$ & $\begin{array}{l}\text { intravaskulär } \\
(\%)\end{array}$ & $\begin{array}{l}\text { Halbwertszeit } \\
\text { (Tage) }\end{array}$ & $\begin{array}{l}\text { Rückverteilung pro Tag } \\
\text { (\%) }\end{array}$ \\
\hline $\lg G_{1,2,4}$ & 12 & 150 & 45 & 22 & 7 \\
\hline $\lg _{3}$ & 0,7 & 150 & 64 & 7 & 17 \\
\hline $\lg M$ & 0,9 & 950 & 78 & 5 & 19 \\
\hline $\lg A$ & 2,5 & 160 & 42 & 6 & 25 \\
\hline $\lg D$ & 0,02 & 175 & 75 & 2,8 & 37 \\
\hline $\lg E$ & 0,0001 & 190 & 45 & 2,5 & 94 \\
\hline Albumin & 45 & 66 & 44 & 17 & 11 \\
\hline Fibrinogen & $3-4$ & 340 & 81 & 4,2 & 24 \\
\hline $\mathrm{C} 3$ & 1,4 & 240 & 67 & 2 & 41 \\
\hline $\mathrm{C} 4$ & 0,5 & 200 & 66 & 2 & 43 \\
\hline
\end{tabular}

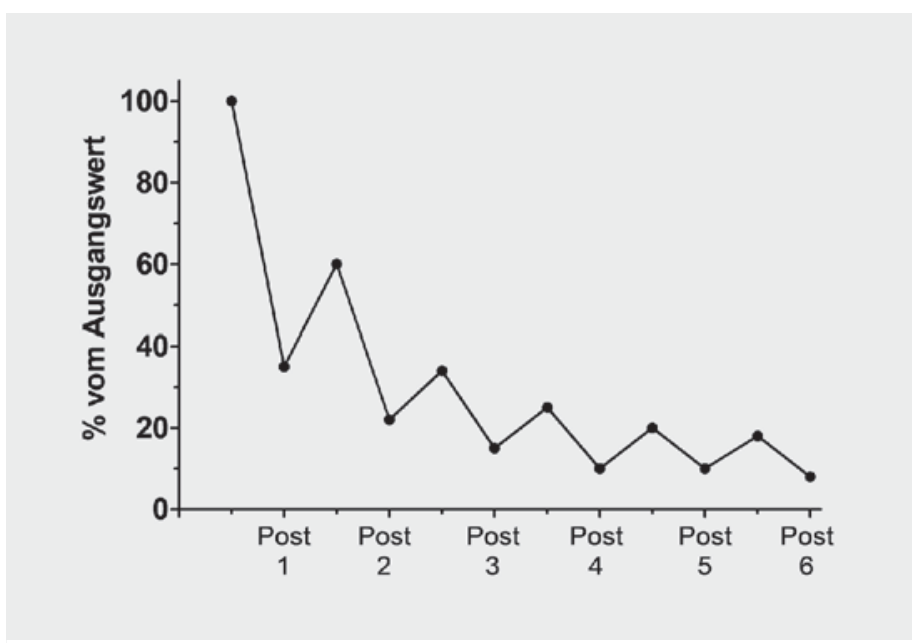

- Abb. 1 Plasmakonzentrationen von Immunglobulin G ( $\mathrm{lgG})$ vor und nach Plasmabehandlungen.

(lgG), verdeutlicht werden. Die intravaskuläre lgG-Konzentration sinkt nach der ersten Apheresebehandlung auf $30 \%$ des Ausgangswertes ab und steigt im Verlauf auf 60-65\% vor der nächsten Apheresebehandlung an. Die erneute Behandlung reduziert die Konzentration dann auf 20-25\%. Hieraus ergibt sich eine Kinetik, bei der nach 5 Apheresebehandlungen die IgG-Plasmakonzentration auf ca. 10\% nach und ca. 20-25\% vor der nächsten Apheresebehandlung vermindert wird ( $>$ Abb. 1). Weitere Plasmabehandlungen in höherer Frequenz zeigen keinen größeren Erfolg, sodass hier Effektivitätsgrenzen erreicht werden.

In \ Tab. 1 sind Charakteristika verschiedener Plasmaproteinen zusammengefasst, die erklären, warum eine medikamentöse immunsuppressive Therapie i. d. R. nur zeitverzögert erfolgreich sein kann. In Abhängigkeit der teilweise sehr langen Halbwertszeit und des Verteilungsvolumens des einzelnen Pathogens kann die Verweildauer nach Unterbindung der Synthese noch sehr lang sein, bei lgG bspw. mehrere Wochen. Das ist eine Begründung für den Einsatz einer TA, mit deren Hilfe eine signifikante Elimination des Pathogens über einen kurzen Zeitraum möglich ist.

\section{Unterschied zwischen TPA und IA}

Der Unterschied zwischen der TPA und der IA liegt in einer gewissen Selektivität der IA. Beim TPA werden die Plasmabestandteile unselektiv entfernt und entweder vollständig durch die Substitution von Fremdplasma oder nur teilweise durch Substitution einer Albumin-Vollelektrolyt-Lösung ersetzt. Die Substitution großer Mengen an Fremdplasma hat ein allergisches Potenzial, wodurch u. a. die Behandlungsvolumina der TPA eingeschränkt sind und i.d. R. bei dem 1-2-fachen Plasmavolumen liegen. Die Verwendung einer Albumin-Vollelektrolyt-Lösung als Substitution resultiert in einer Verminderung der Plasmakonzentration u.a. der Gerinnungsfaktoren und der Ig, die im Verlauf der Therapie substituiert werden zu müssen. Im Rahmen der IA werden mit einer gewissen Selektivität vor allem AK und AK-Komplexe aus dem Plasma entfernt. Dabei ist zu bedenken, dass neben Ig auch andere Plasmabestandteile, wie bspw. Fibrinogen, entfernt werden, was einen Einfluss auf die Gerinnung hat. Des Weiteren gibt es unterschiedliche Affinitäten der einzelnen Immunadsorptionssäulen bspw. bzgl. der Bindung der lgG-Subtypen und der Regenerierbarkeit der eingesetzten Säulen.

\section{Indikationen für eine TPA und IA}

In vielen Bereichen der Medizin werden TPA und IA erfolgreich eingesetzt. In den Leitlinien der „American Society of Apheresis“ wurden die Empfehlungen zur Durchführung einer Apheresetherapie 2019 aktualisiert, in der die Behandlung von 84 Erkrankungen und 157 Indikationen systematisch kategorisiert wurden [5]. Die Einteilung der Empfehlungen erfolgt weiterhin nach 4 Kategorien ( $\triangleright$ Tab. 2). Dabei fallen aktuell 29 Indikationen in die 
- Tab. 2 Indikationsstellungen nach Leitlinien der „American Society of Apheresis“ aus dem Jahr 2019 [5].

\begin{tabular}{|l|l|}
\hline Kategorie & Beschreibung \\
\hline I & $\begin{array}{l}\text { Erkrankungen, bei denen die Apherese entweder als alleinige Therapie oder in Verbindung mit einem anderen Therapie- } \\
\text { konzept eine akzeptiertes Primärtherapie darstellt }\end{array}$ \\
\hline II & $\begin{array}{l}\text { Erkrankungen, bei denen die Apherese entweder als alleinige Therapie oder in Verbindung mit einem anderen Therapie- } \\
\text { konzept eine akzeptierte Sekundärtherapie darstellt }\end{array}$ \\
\hline III & Erkrankungen, bei denen die Apherese in individuellen klinischen Situationen eine Therapiealternative darstellen kann \\
\hline IV & Erkrankungen, bei denen die Apherese ineffektiv oder gefährlich ist \\
\hline
\end{tabular}

Kategorie I, 43 in die Kategorie II, 79 in die Kategorie III und 6 in die Kategorie IV.

Im Rahmen der Leitlinie werden die in $>$ Tab. $\mathbf{3}$ wiedergegebenen Kriterien für die jeweiligen Empfehlungsgrade verwendet [5].

- Tab. 4 fasst wichtige therapeutische Einsatzgebiete der TA zusammen, von denen im Folgenden wichtige nephrologische und neurologische Erkrankungen dargestellt werden.

Insgesamt ist die Datenlage für viele der möglichen Einsatzgebiete einer TA begrenzt, da prospektive und multizentrische Studien mit ausreichender Power für viele Indikationsgebiete fehlen. Dennoch konnte bei vielen Erkrankungen durch größere Fallstudien die Bedeutung der TA gezeigt werden. Insbesondere bei Auto-AK-assoziierten Erkrankungsbildern stellt die IA eine Alternative zum TPA dar. Vergleichende Studien zwischen dem Erfolg eines TPA und einer IA stellen Ausnahmen dar und umfassen dann nur geringe Fallzahlen.

\section{Nephrologische Indikationen}

In vielen Bereichen der Nephrologie wird die TA eingesetzt. Nach Daten der Canadian Apheresis Group aus dem Jahr 2014 waren die häufigsten renalen Diagnosen, die zu einem TA führten, die TMA (56\% TTP, 3\% aHUS, 3 \% HUS), transplantationsassoziierte Indikationen (8,4\% humorale Rejektion, 1,9\% Desensitisierung, 1,6\% AB0-inkompatible Transplantation), eine AASV (7,5\%), eine Kryoglobulinämie (6,7\%), eine FSGS (6,5\%) und ein Goodpasture-Syndrom (5,3\%) [7]. Im Folgenden soll auf einige der wichtigsten Indikationen kurz eingegangen werden.

\section{Thrombotische Mikroangiopathie (TMA)}

Eine TMA kann durch viele unterschiedliche Mechanismen ausgelöst werden. Hierzu zählt bspw. eine Infektion mit Shiga-Toxin bildende E. coli 0157:H7 (STEC), die zur Ausbildung des hämolytisch-urämischen Syndroms (STEC-HUS) führen. Daneben stellen autoimmunologische oder genetische Veränderungen von Faktoren der Gerinnung (u. a. THBD, DGKE, PLG) oder des Komplementsystems (u. a.
- Tab. 3 Grad der Empfehlung nach dem „Grading of Recommendations Assessment, Development and Evaluation (GRADE)“ System [6].

\begin{tabular}{|c|l|}
\hline $\begin{array}{l}\text { Grad der } \\
\text { Empfehlung }\end{array}$ & Beschreibung \\
\hline Grad 1A & $\begin{array}{l}\text { starke Empfehlung, } \\
\text { hohe Qualität der Evidenz }\end{array}$ \\
\hline Grad 1B & $\begin{array}{l}\text { starke Empfehlung, } \\
\text { mäßige Qualität der Evidenz }\end{array}$ \\
\hline Grad 1C & $\begin{array}{l}\text { starke Empfehlung, } \\
\text { (sehr) niedrige Qualität der Evidenz }\end{array}$ \\
\hline Grad 2A & $\begin{array}{l}\text { schwache Empfehlung, } \\
\text { hohe Qualität der Evidenz }\end{array}$ \\
\hline Grad 2B & $\begin{array}{l}\text { schwache Empfehlung, } \\
\text { mäßige Qualität der Evidenz }\end{array}$ \\
\hline Grad 2C & $\begin{array}{l}\text { schwache Empfehlung, } \\
\text { (sehr) niedrige Qualität der Evidenz }\end{array}$ \\
\hline
\end{tabular}

Faktor H) Gründe für die Ausbildung eines atypischen HUS (aHUS) dar. Transplantationsassoziierte TMA-Formen basieren entweder auf Nebenwirkungen einer HochdosisChemotherapie, einer Bestrahlung, einer Infektion oder einer Graft-versus-Host-Reaktion. Weitere Auslöser können Medikamente, wie bspw. Calcineurininhibitoren oder Ticlopidin, oder Autoimmunerkrankungen, wie bspw. die Sklerodermie, oder eine maligne Hypertonie sein. Eine weitere klinisch eindrückliche Erkrankung ist die thrombotisch-thrombozytopenische Purpura (TTP), die am häufigsten erworben durch die Ausbildung von Auto-AK gegen ADAMTS13 (, a disintegrin and metalloproteinase with a thrombospondin type 1 motif, member 13“) entsteht. Eine eindeutige klinische Differenzierung zwischen diesen Formen der TMA ist im akuten Stadium nicht immer exakt möglich.

\section{Erworbene thrombotisch-thrombozyto- penische Purpura (TTP)}

Die klinischen Symptome einer TTP sind eine Coombs negative, mikroangiopathische hämolytische Anämie und eine ausgeprägte Thrombopenie, die in der Mehrzahl der Fälle mit einer neurologischen und gelegentlich renalen Symptomatik verbunden ist. Der unbehandelte Verlauf 
- Tab.4 Wichtige Indikationen therapeutischer Aphereseverfahren nach den Leitlinien des ASFA 2019 (Fortsetzung siehe folgende Seite) [5].

\begin{tabular}{|c|c|c|c|c|c|}
\hline Bereich & Erkrankung & Modalität & Besonderheit & Kategorie & Grad \\
\hline \multirow[t]{31}{*}{ Nephrologie } & \multirow[t]{4}{*}{ ANCA-assoziierte Vaskulitiden (AASV) } & TPA & $\begin{array}{l}\text { MPA/GPA/RLV: RPGN, } \\
{[\text { Kreatinin }]_{\text {Plasma }}>5,7 \mathrm{mg} / \mathrm{dl}}\end{array}$ & 1 & $1 \mathrm{~A}$ \\
\hline & & TPA & $\begin{array}{l}\text { MPA/GPA/RLV: RPGN, } \\
{[\text { Kreatinin }]_{\text {Plasma }}<5,7 \mathrm{mg} / \mathrm{dl}}\end{array}$ & III & $2 C$ \\
\hline & & TPA & MPA/GPA/RLV: DAH & 1 & $1 \mathrm{C}$ \\
\hline & & TPA & EGPA & III & $2 \mathrm{C}$ \\
\hline & \multirow{3}{*}{$\begin{array}{l}\text { Anti-glomeruläre-Basalmembran-Syndrom } \\
\text { (Goodpasture-Syndrom) }\end{array}$} & TPE & diffuse alveoläre Hämorrhagie (DAH) & 1 & $1 \mathrm{C}$ \\
\hline & & TPE & dialyseunabhängig & 1 & 1B \\
\hline & & TPE & dialysepflichtig ohne DAH & III & $2 B$ \\
\hline & \multirow[t]{2}{*}{$\begin{array}{l}\text { blutgruppenungleiche Nierentransplan- } \\
\text { tation }\end{array}$} & TPA/IA & $\begin{array}{l}\text { Desensitisierung des Empfängers (Lebend- } \\
\text { spende) }\end{array}$ & 1 & 1B \\
\hline & & TPA/IA & humorale Rejektion & II & 1B \\
\hline & \multirow[t]{3}{*}{ blutgruppengleiche Nierentransplantation } & TPA/IA & $\begin{array}{l}\text { Desensitisierung des Empfängers (Lebend- } \\
\text { spende) }\end{array}$ & 1 & 1B \\
\hline & & TPA/IA & $\begin{array}{l}\text { Desensitisierung des Empfängers (postmortale } \\
\text { Spende) }\end{array}$ & III & $2 C$ \\
\hline & & TPA/IA & humorale Rejektion & 1 & 1B \\
\hline & $\begin{array}{l}\text { "catastrophic antiphospholipid syndrome“ } \\
\text { (CAPS) }\end{array}$ & TPA & & 1 & $2 C$ \\
\hline & \multirow[t]{2}{*}{ Cyroglobulinämie } & TPA & schwere Verlaufsform & II & $2 \mathrm{~A}$ \\
\hline & & IA & schwere Verlaufsform & ॥ & $2 B$ \\
\hline & \multirow{2}{*}{$\begin{array}{l}\text { fokal-segmentale Glomerulosklerose } \\
\text { (FSGS) }\end{array}$} & TPA/IA & Rekurrenz nach Transplantation & 1 & 1B \\
\hline & & TPA & steroidresistent in den Eigennieren & III & $2 C$ \\
\hline & \multirow[t]{2}{*}{ IgA-Vaskulitis (Purpura Henoch-Schönlein) } & TPA & RPGN & III & $2 C$ \\
\hline & & TPA & schwere extrarenale Manifestation & III & $2 C$ \\
\hline & $\begin{array}{l}\text { thrombotische Mikroangiopathie (TMA), } \\
\text { Infektion }\end{array}$ & TPA/IA & STEC-HUS, schwerwiegend & III & $2 C$ \\
\hline & \multirow{3}{*}{$\begin{array}{l}\text { Hyperviskositätssyndrom bei } \\
\text { Hypergammaglobulinämie }\end{array}$} & TPA & symptomatisch & 1 & 1B \\
\hline & & \multirow[t]{2}{*}{ TPA } & prophylaktisch vor Rituximab & 1 & $1 \mathrm{C}$ \\
\hline & & & spHUS & III & $2 \mathrm{C}$ \\
\hline & TMA, Koagulation & TPA & Mutation THBD, DGKE oder PLG & III & $2 \mathrm{C}$ \\
\hline & \multirow[t]{5}{*}{ TMA, Komplement } & TPA & Faktor H Auto-AK & 1 & $2 C$ \\
\hline & & TPA & Mutation Komplementfaktoren & III & $2 C$ \\
\hline & & TPA & Ticlopidin & 1 & 2B \\
\hline & & TPA & Clopidogrel & III & $2 \mathrm{~B}$ \\
\hline & & TPA & Gemcitabin & IV & $2 C$ \\
\hline & $\begin{array}{l}\text { TMA, thrombotisch-thrombozytopenische } \\
\text { Purpura (TTP) }\end{array}$ & TPA & & 1 & IA \\
\hline & systemischer Lupus erythematodes & TPA & schwerwiegender systemischer Verlauf & II & $2 C$ \\
\hline
\end{tabular}

einer TTP ist in den allermeisten Fällen (80-90\%) tödlich. Nach dem Ausschluss anderer wichtiger Differenzialdiagnosen sollte eine TA innerhalb weniger Stunden nach Beginn der klinischen Symptomatik gestartet werden. Für die klinische Einschätzung kann der PLASMIC-Score genutzt werden [8]. Pathophysiologisch ist das Enzym ADAMTS13 von großer Bedeutung, das durch Abbau endothelial sezernierter großer Multimere eine normale Zusammensetzung der vWF-Multimere (vWF: von Willebrand Faktor) bewirkt [9].
Zur Sicherung der klinisch gestellten Diagnose sollte die Bestimmung der Aktivität der ADAMTS13 und die Bestimmung von Auto-AK gegen ADAMTS13 erfolgen. Die Au-

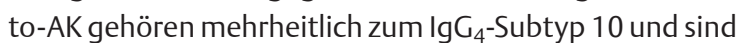
im Rahmen der idiopathischen TTP in der Mehrzahl nachweisbar $[10,11]$. Hier ist besonders darauf zu achten, dass die für diese Bestimmung notwendigen Blutentnahmen vor dem Beginn der ersten TA erfolgen, um falsch negative Befunde zu vermeiden. 
- Tab. 4 Fortsetzung.

\begin{tabular}{|c|c|c|c|c|c|}
\hline Bereich & Erkrankung & Modalität & Besonderheit & Kategorie & Grad \\
\hline \multirow[t]{18}{*}{ Neurologie } & $\begin{array}{l}\text { akute disseminierte Enzephalomyelitis } \\
\text { (ADEM) }\end{array}$ & TPA & steroidrefraktärer Verlauf & II & $2 \mathrm{C}$ \\
\hline & \multirow{2}{*}{$\begin{array}{l}\text { akute inflammatorische demyelinisierende } \\
\text { Polyradikuloneuropathie (AIDP, Guil- } \\
\text { lain-Barré-Syndrom) }\end{array}$} & TPA & Primärtherapie & 1 & $1 \mathrm{~A}$ \\
\hline & & TPA & Erhaltungstherapie & III & $2 C$ \\
\hline & $\begin{array}{l}\text { chronische inflammatorische demyelinisie- } \\
\text { rende Polyradikuloneuropathie (CIDP) }\end{array}$ & TPA/IA & & 1 & 1B \\
\hline & $\begin{array}{l}\text { Lambert-Eaton-Myasthenie-Syndrom } \\
\text { (LEMS) }\end{array}$ & TPA & & II & $2 \mathrm{C}$ \\
\hline & \multirow[t]{4}{*}{ Multiple Sklerose (MS) } & TPA & akute Erkrankung/Relaps & II & $1 \mathrm{~A}$ \\
\hline & & IA & akute Erkrankung/Relaps & II & 1B \\
\hline & & TPA & chronische Erkrankung & III & $2 \mathrm{~B}$ \\
\hline & & $\mathrm{IA}$ & chronische Erkrankung & III & $2 \mathrm{~B}$ \\
\hline & \multirow[t]{2}{*}{ Myasthenia gravis (MG) } & TPA/IA & akute, kurzfristige Therapie & 1 & 1B \\
\hline & & TPA/IA & langfristige Therapie & II & 2B \\
\hline & \multirow{2}{*}{$\begin{array}{l}\text { Neuromyelitis optica Spektrumerkrankun- } \\
\text { gen (NMOSD) }\end{array}$} & TPA & akute Erkrankung/Relaps & II & 1B \\
\hline & & $\mathrm{IA}$ & akute Erkrankung/Relaps & II & $1 \mathrm{C}$ \\
\hline & $\begin{array}{l}\text { N-Methyl-D-Aspertat (NMDA) Rezeptor AK } \\
\text { Enzephalitis }\end{array}$ & TPA/IA & & 1 & $1 \mathrm{C}$ \\
\hline & \multirow{4}{*}{$\begin{array}{l}\text { paraproteinämische demyelinisierende } \\
\text { Neuropathie, chronisch erworbene demye- } \\
\text { linisierende Polyneuropathie }\end{array}$} & TPA & $\lg G / \lg A / I G M$ & I & 1B \\
\hline & & TPA & Anti-MAG-Neuropathie & III & $1 \mathrm{C}$ \\
\hline & & TPA & multiples Myelom & III & $2 \mathrm{C}$ \\
\hline & & TPA & multifokale motorische Neuropathie & IV & $1 \mathrm{C}$ \\
\hline
\end{tabular}

Die Therapie der TTP ist die PS, wodurch die Letalität dieser Erkrankung stark gesunken ist (10-20\%) [12]. Eine Alternative stellt die Plasmainfusion da, die allerdings aufgrund des benötigten Volumens in der akuten Situation nur eingeschränkt genutzt werden kann. Durch die Plasmaseparation werden Auto-AK entfernt und die Plasmaaktivität der ADAMTS13 durch Substitution erhöht. Die PS sollte initial täglich erfolgen, ein Behandlungsvolumen von 1-1,5 Plasmavolumina umfassen und i. d. R. bis zur Normalisierung der Thrombozytenzahl im Blut (> $\left.150 \times 10^{9} / \mathrm{I}\right)$ und Normalisierung der LDH-Plasmakonzentration erfolgen. Im Anschluss daran ist individuell zu entscheiden, ob man die PS beendet oder umtägig weiterführt. Eine therapeutische Alternative stellt Caplacizumab dar. Hierbei handelt es sich um einen SingleDomain-AK gegen die Thrombozyten bindende Domäne des vWF. In einer Phase-II- und einer Phase-III-Studie zeigte sich unter Caplacizumab eine schnellere Remission unter einer verminderten Anzahl an PS.

\section{Nierentransplantation}

\section{Blutgruppenungleiche Nierentransplantation}

Im Rahmen einer blutgruppenungleichen Nierentransplantation kann durch die Anwendung einer TA die Grundvoraussetzung für einen erfolgreiche Verlauf geschaffen werden. Im Blut des potenziellen Empfängers finden sich, mit Ausnahme von Patienten der Blutgruppe AB, AK gegen an- dere Blutgruppenantigene, die sog. Isoagglutinine, durch die akute oder hyperakute Rejektionen ausgelöst werden können. Mithilfe des TPA, einer selektiven [13] oder unselektiven IA [14] oder einer Kombination aus unterschiedlichen Verfahren [15], können die Isoagglutinine des Empfängers, die sich gegen die Blutgruppe des Spenders richten, in der Mehrzahl der Fälle ausreichend reduziert werden, wodurch eine blutgruppenungleiche Transplantation möglich wird. Bei humoralen Rejektionen zeigt der Einsatz einer TA, insbesondere der PP und der IA, ebenfalls einen zusätzlichen Nutzen [5].

\section{Blutgruppengleiche Nierentransplantation}

Im Rahmen blutgruppengleicher Nierentransplantationen spielen TA eine wichtige Rolle. Vergleichbar mit der Situation bei der blutgruppenungleichen Nierentransplantation können sowohl die PP [16] als auch die IA [17] erfolgreich im Bereich der humoralen Rejektion eingesetzt werden, die insbesondere durch donorspezifische HLAAK (DSA) ausgelöst wird. Durch die Kombination aus TA und immunsuppressiver Therapie (IVIG, Rituximab) kann das Transplantatüberleben nach Rejektionsbehandlung auf bis zu $90 \%$ gesteigert werden. Neben diesem Einsatz werden die PP und die IA auch zur Vorbereitung von Lebendspenden genutzt, wenn der Empfänger bereits vor der Lebendspende präformierte DSA gebildet hat. Im Rahmen von unterschiedlichen Desensitisierungsprotokollen 
kann eine Lebendspende dadurch in erfahrenen Transplantationszentren möglich gemacht werden [18].

\section{ANCA-assoziierte Vaskulitiden (AASV)}

Die nekrotisierenden „antineutrophile zytoplasmatische AK“ (ANCA) assoziierten Kleingefäßvaskulitiden (AASV: „ANCA-associated small vasculitis“) stellen wichtige Ursachen für eine rapid-progrediente Glomerulonephritis (RPGN) dar. Aufgrund der histologisch lediglich minimalen bis fehlenden Immunkomplex-Ablagerungen wird diese Form als pauci-immun bezeichnet. Zu den AASV gehören die „Granulomatose mit Polyangiitis“ (GPA), die „mikroskopische Polyangiitis“ (MPA) und die „eosinophile Granulomatose mit Polyangiitis“ (EGPA), wobei Überlappungen zwischen diesen Formen vorkommen. Die AASV gehören ebenfalls in die Klasse der pulmorenalen Syndrome, da vergleichbar zum Goodpasture-Syndrom, neben der RPGN, auch diffus-alveoläre Hämorrhagien (DAH) auftreten können. Das klinische Spektrum der einzelnen AASV-Unterformen reicht von ausschließlich renal bis ausgeprägt systemisch. Im Vergleich zum Goodpasture-Syndrom ist der renale Verlaufi. d. R. langsamer und die GFR-Abnahme unbehandelt teilweise über Wochen progredient. Es kommen aber auch zeitlich rasch progrediente Verlaufsformen vor, wobei teilweise parallel Anti-GBM-AK vorliegen. Neben der Immunsuppression, die zumeist aus der Kombination Kortison mit Cyclophosphamid oder Rituximab besteht, ist die PP (ohne DAH) oder die PS (mit DAH) eine therapeutische Option. Die pathophysiologisch relevanten ANCA-Auto-AK besitzen ein geringes Verteilungsvolumen, eine geringe Umverteilungsrate in den intravaskulären Raum und eine lange Halbwertzeit, sodass die Zeit bis zum Therapieerfolg durch eine alleinige medikamentöse Immunsuppression verzögern einsetzen kann.

In der MEPEX-Studie wurde bei 137 Patienten mit AASV und fortgeschrittener Niereninsuffizienz (Kreatinin $>5,7 \mathrm{mg} / \mathrm{dl}$ inkl. Dialyse) gezeigt, dass die Durchführung von Plasmapheresen im Vergleich zu einer Kortisonstoßtherapie ( $3 \mathrm{~g}$ ) zusätzlich zu einer oralen Therapie mit Cyclophosphamid und Kortison bei Patienten mit fortgeschrittener Niereninsuffizienz nach 3 und 12 Monaten mit einem reduzierten Risiko für eine Dialysepflichtigkeit verbunden war, wobei das Patientenüberleben und die Rate schwerer Nebenwirkungen in beiden Gruppen vergleichbar war [19]. Bei initial dialysepflichtiger Niereninsuffizienz zeigt sich ein Vorteil für die TA in Bezug auf die Verbesserung der Nierenfunktion mit Beendigung der Dialysebehandlung im Verlauf [20]. Es ergibt sich somit eine starke Empfehlung für den Einsatz der TA bei Patienten mit fortgeschrittener bis dialysepflichtiger Niereninsuffizienz (Kreatinin > 5,7 mg/dl), schwerer DAH und kombiniertem Nachweis von ANCA- und Anti-GBM-Auto-AK [5]. Die Daten der PEXIVAS-Studie, in der über 700 Patienten mit GPA oder MPA mit schwerer Niereninsuffizienz (GFR $<50 \mathrm{ml} / \mathrm{min}$ pro $1,73 \mathrm{~m}^{2}$ ) oder DAH eingeschlossen wurden, werden weitere wichtige Anhaltspunkte über den
Stellenwert des TPA geben. In der Behandlung der AASV konnte neben der TPA auch die IA erfolgreich eingesetzt werden [21-23].

\section{Goodpasture-Syndrom}

Das Goodpasture-Syndrom ist ebenfalls eine Erkrankung aus dem Formenkreis der pulmorenalen Syndrome. Es ist Ursache einer RPGN, die mit DAH assoziiert ist und eine hohe Assoziation zu Polymorphismen der MHC-Klasse-IIGene, insbesondere HLA-DRB1, aufweist. Bis zu 40 \% der Betroffenen zeigen eine alleinige renale Manifestation. Zu beachten ist auch, dass regelmäßig pulmonale Symptome vor typischen renalen Symptomen auftreten können. Bei allen DAH ist daher u. a. neben der Differenzialdiagnose einer AASV das Goodpasture-Syndrom immer auszuschließen. Diagnostisch relevant ist der Nachweis von Anti-GBMAuto-AK, die sich gegen die $\alpha 3$-Kette des Typ-IV-Kollagens richten. Dieser Kollagentyp findet sich sowohl in der Basalmembran der Niere als auch der Lunge.

Aufgrund der erheblichen Bedeutung für die Langzeitprognose ist neben der raschen und suffizienten Diagnostik der unmittelbare Beginn einer adäquaten Therapie von großer Bedeutung. Aufgrund der pathophysiologischen Bedeutung der Anti-GBM ist neben einer immunsuppressiven Therapie, bestehend i. d. R. aus Cyclophosphamid und Kortison, der frühzeitige Beginn einer PP (ohne DAH) oder einer PS (mit DAH) von großer Bedeutung, um die Menge der Anti-GBM-AK rasch zu vermindern. Da fortgeschrittene renale Folgen eines Goodpasture-Syndroms häufig irreversibel sind, bestehen unterschiedliche Empfehlungen für den Einsatz der TA. Im Rahmen eines akuten, nicht dialysepflichtigen Nierenversagens und in der Situation einer DAH besteht eine starke Empfehlung für einen sofortigen Beginn der TA [5]. Bei Patienten mit einem akuten, dialysepflichtigen Nierenversagen ist die Wahrscheinlichkeit einer Verbesserung durch eine TA nicht erwiesen, sodass hier nur eine klare Empfehlung des Einsatzes bei gleichzeitigem Vorliegen einer DAH vorliegt [5]. In Fällen eines dialysepflichtigen Nierenversagens ohne DAH ist die Indikation zur TA individuell zu treffen [5]. Neben dem TPA konnte auch die IA erfolgreich beim Goodpasture-Syndrom angewendet werden.

\section{Kryoglobulinämie}

Eine Kryoglobulinämie ist assoziiert mit vielen Erkrankungen, bspw. viralen Infektionen (Hepatitis B, Hepatitis C), Autoimmunerkrankungen oder lymphoproliferativen Erkrankungen. Die Schwere der klinischen Symptome reicht von milden (u.a. Purpura, Neuropathie) bis sehr schweren (u. a. RPGN) Verläufen. Die Zusammensetzung der Kryoglobuline kann in 3 unterschiedliche Typen unterteilt werden. Beim Typ I sind monoklonale Immunglobuline bspw. im Rahmen eines Plasmozytoms (lgG) oder eines Morbus Waldenström (IوM) vorhanden. Beim Typ II und III liegt jeweils eine gemischte Kryoglobulinämie vor, die beim Typ II aus polyklonalen IgGs als Antigen und monoklonalen 
IgMs als Auto-AK und beim Typ III aus polyklonalen IgGs und polyklonalen IgMs besteht. Bis zu $90 \%$ der Patienten mit gemischten Kryoglobulinen sind Hepatitis V positiv. Neben der immunsuppressiven und antiviralen Therapie stellt die TA einen zusätzlichen Therapieansatz bei schweren Verlaufsformen aufgrund der effektiven Elimination verschiedener Kryoglobuline dar. Eine Alternative stellt die Doppelfiltrationsplasmapherese (DFPP) dar.

\section{Rekurrente fokal-segmentale Glomerulo- sklerose (FSGS)}

Die Diagnose der FSGS stützt sich auf das histopathologische Muster in der Nierenbiopsie. Es können unterschiedliche Formen der FSGS („cellular“, „collapsing“, „tip lesion“, „perihilar“, „,not specified“) unterschieden werden. $80 \%$ aller FSGS-Formen sind idiopathisch, deren pathophysiologische Ursache ein unbekannter Plasmafaktor oder mehrere unbekannte Plasmafaktoren mit einem vermuteten Molekulargewicht zwischen 30 und 50 kDa ist. Die Permeabilität der glomerulären Barriere wird dahingehend beeinflusst, dass u. a. eine nephrotische Proteinurie entsteht.

Patienten mit einer primären FSGS der Eigennieren, die eine Proteinurie von 3-3,5 g/Tag aufweisen, scheinen nicht von einer TA zu profitieren, sodass hier die initiale immunsuppressive Therapie mit Kortison Standard ist. Beim Versagen einer immunsuppressiven Therapie stellt sowohl der TPA (i. d. R. als PP) als auch die IA eine therapeutische Alternative dar. Interessanterweise profitieren nierentransplantierte Patienten im Rahmen eines Rezidivs in der Transplantatniere von einem TPA oder einer IA zusätzlich zu Kortison und ggf. der Umstellung der Immunsuppression. Mit der PP/IA sollte direkt nach Diagnosesicherung der Rekurrenz begonnen werden, da ein verzögerter Beginn mit einer schlechteren Remissionsrate assoziiert sein kann. Die Anzahl der TA und die Behandlungsdauer sind individuell festzulegen, da eine Remissionsrate von bis zu 70 \% möglich ist. Des Weiteren gibt es bislang keine guten klinischen oder laborchemischen Parameter, an denen sich der Erfolg einer PP prognostizieren lässt [5]. Das Ausmaß der Proteinurie stellt einen möglichen Verlaufsparameter dar. Die Tatsache, dass eine IA zu einer Rekurrenz der FSGS beitragen kann [24-26], belegt, dass es sich bei den Plasmafaktoren um Immunglobulin ähnliche Mediatoren handeln könnte. Interessanterweise wird die Plasmakonzentration des löslichen UrokinasePlasminogen-Aktivator-Rezeptors (suPAR) nicht durch die IA reduziert [27].

\section{Neurologische Indikationen}

In den letzten Jahren hat die TA auch einen festen Platz in der Behandlung neurologischer Erkrankungen eingenommen. Die Myasthenia gravis stellt ein Musterbeispiel einer neurologischen Erkrankung dar, die durch eine TA erfolgreich therapiert werden kann. In den letzten Jahren wurde die pathophysiologische Bedeutung von Auto-AK bei einer \ab.5 Übersicht über wichtige Indikationen für eine Plasmatherapie in der Neurologie.

\begin{tabular}{|c|c|}
\hline Kategorie & Erkrankung \\
\hline \multirow{4}{*}{$\begin{array}{l}\text { Erkrankungen des } \\
\text { zentralen Nervensystems }\end{array}$} & akute disseminierte Enzephalomyelitis (ADEM) \\
\hline & Multiple Sklerose (MS) \\
\hline & Neuromyelitis optica (NMO) \\
\hline & $\begin{array}{l}\text { Antikörper assoziierte limbische Enzephalitis } \\
\text { (LE) }\end{array}$ \\
\hline \multirow[t]{2}{*}{$\begin{array}{l}\text { Erkrankungen des } \\
\text { peripheren Nervensystems }\end{array}$} & $\begin{array}{l}\text { akute inflammatorische demyelinisierende } \\
\text { Polyradiculoneuropathien (AIDP) }\end{array}$ \\
\hline & $\begin{array}{l}\text { chronische inflammatorische demyelinisierende } \\
\text { Polyradiculoneuropathien (CIDP) }\end{array}$ \\
\hline \multirow{3}{*}{$\begin{array}{l}\text { neuromuskuläre } \\
\text { Erkrankungen }\end{array}$} & Myasthenia gravis (MG) \\
\hline & Lambert-Eaton-Myasthenie-Syndrom (LEMS) \\
\hline & Neuromyotonia (NM) \\
\hline
\end{tabular}

Vielzahl neurologischer Erkrankungen nachgewiesen. In - Tab. $\mathbf{5}$ sind typische neurologische Indikationen für eine TA zusammengefasst.

\section{Akute disseminierte Enzephalomyelitis (ADEM)}

Die ADEM ist eine akute inflammatorische demyelinisierende Erkrankung des zentralen Nervensystems (ZNS), die hauptsächlich die zentralnervöse weiße Substanz des Gehirns und des Rückenmarks betrifft [35, 36]. Auslöser sind häufig virale/bakterielle Infektionen oder Impfungen. Pathophysiologisch erscheint eine autoimmunlogische Reaktion gegen Myelin oder andere Autoantigene vorstellbar [42]. Die Erkrankung tritt innerhalb von Tagen bis einigen Wochen nach dem auslösenden Ereignis mit einer akuten Enzephalitis und multifokalen neurologischen Symptomen auf, verläuft i.d. R. ohne Rezidive und zeigt eine günstige Prognose mit vollständiger klinischer Remission nach Wochen bis Monaten in 55-95\% der Fälle. Allerdings können auch letale Verläufe vorkommen. Die Abgrenzung zwischen einer ADEM und der ersten Manifestation einer Multiplen Sklerose (MS) ist wichtig und u. U. schwierig. Das Vorhandensein von unterschiedlichen neurologischen Symptomen, das Fehlen oligoklonaler Banden im Liquor und pathologischer Zeichen im MRT in der Rekonvaleszenz deuten auf eine ADEM hin.

Die Gabe von hochdosierten Steroiden stellt die Initialtherapie dar. Der Stellenwert der TPA (i.d. R. PP) ergibt sich aus der möglichen autoimmunologisch vermittelten Inflammation und kommt insbesondere für schwere und therapieresistente Verlaufsformen in Betracht. Hierdurch kann eine kurzfristige Verbesserung der klinischen Symptome in bis zu $40 \%$ erzielt werden [37]. Wenn eine klinische Besserung nicht nach den ersten 3-5 PP eingetreten ist, ist eine Verbesserung der neurologischen Symptome durch Fortführung der Behandlung nicht zu erwarten [37]. 


\section{Multiple Sklerose (MS)}

Die MS ist eine chronisch-inflammatorische Erkrankung des ZNS, bei der die Demyelinisierung der Nervenfasern das pathophysiologische Ereignis darstellt. Der klinische Verlauf der Erkrankung kann in eine benigne, eine schubförmig remittierende (RRMS), eine primär oder sekundär progrediente Form eingeteilt werden. Bei bis zu $15 \%$ aller Patienten zeigt sich eine primär progressive Form, wobei viele MS-Patienten nach 10-20 Jahren eine sekundär progressive Form aufzeigen. Bei Rezidiven können nahezu alle ZNS-Bereiche betroffen sein. Bei der Mehrheit dieser Patienten treten Rezidive im zeitlichen Verlauf zunehmend häufiger auf und dauern immer länger an. Die Folge ist, dass sich die körperlichen Einschränkungen der Patienten durch die neurologischen Schädigungen zwischen den einzelnen Rezidiven zunehmend verschlechtern und der Krankheitsverlauf über Jahre progredient ist. Die pathologischen Veränderungen stellen sich als entzündliche Veränderungen im Bereich des Gehirns und des Rückenmarks dar, die zu einer zunehmenden Demyelinisierung und einer Gliose führen. Innerhalb der Pathophysiologie zeigen sich unterschiedliche autoimmunologische Phänomene, inkl. zellulärer (T-Zell- und Makrophagen-Aktivierung) und humoraler (autoreaktiver AK-)Komponenten [38-40]. Die MS kann in 4 unterschiedliche histopathologische Unterformen eingeteilt werden ( $\bullet$ Tab. 6) [41].

Ein zentraler Mechanismus innerhalb der heterogenen Pathophysiologie der MS scheint B-Zell-vermittelt zu sein. Hierzu zählt neben der Produktion von autoreaktiven AK, die Aktivierung von T-Zellen durch die Antigen-Präsentation und die Produktion von Zytokinen [40]. Daher sind wichtige etablierte Therapien auf die Modulation von B-Zellen ausgerichtet [42, 43]. Als mögliche Angriffspunkte für Auto-AK wurden das Myelin-Oligodendrozyten-Glykoprotein (MOG) oder das myelinbasische Protein (MBP) beschrieben. Auto-AK gegen diese Antigene wurden im Blut und in der Zerebrospinalflüssigkeit von MSPatienten beschrieben [44, 45]. Die pathophysiologische Bedeutung dieser Auto-AK konnte aber bislang nicht validiert werden $[46,47]$.

Die TA stellt in diesem Zusammenhang einen wichtigen therapeutischen Ansatz dar, wobei sich sowohl die PP als auch die IA als effektive Therapie herausgestellt haben [34, 41, 48-51]. In einer Placebo kontrollierten Cross-Over-Studie zeigte sich, dass durch die PP eine funktionelle Verbesserung bei Patienten mit steroidrefraktären Rezidiven einer akut inflammatorischen Demyelinisierung [49] und bei Patienten mit steroidrefraktären Rezidiven einer sekundär progressiven Verlaufsform erreicht werden konnte [52]. Hier scheint es von großer Bedeutung zu sein, welcher MS-Typ vorliegt. Die PP konnte insbesondere bei Patienten mit MS Typ 2, also dem AK- und Komplement vermittelten Typ, erfolgreich eingesetzt werden, wohingegen beim Typ 1 und 3 keine nennenswerten Erfolge erzielt werden konnten. Insbesondere im Bereich der therapie-
- Tab. 6 Einteilung der Multiplen Sklerose (MS) nach histopathologischen Kriterien.

\begin{tabular}{|c|c|}
\hline Typ & Beschreibung \\
\hline 1 & Infiltration mit T-Zellen und Makrophagen \\
\hline 2 & $\begin{array}{l}\text { Antikörper und Komplement vermittelte Demyelini- } \\
\text { sierung }\end{array}$ \\
\hline 3 & distale Oligodendrogliopathie \\
\hline 4 & $\begin{array}{l}\text { Oligodendrozytendegeneration in der die Plaques } \\
\text { umgebenden weißen Substanz }\end{array}$ \\
\hline
\end{tabular}

refraktären MS besteht auch im Rahmen nationaler und internationaler Leitlinien die Empfehlung der Durchführung einer PP oder einer IA [43, 53]. Zusammengefasst zeigten bis zu $76 \%$ von 128 Patienten in 3 Studien eine deutliche funktionelle Verbesserung über einen Zeitraum von 6-12 Monaten, nachdem diese mit PP behandelt wurden. Hierbei kam es im Durchschnitt nach 3 PP zu einer signifikanten klinischen Besserung $[50,54,55]$.

In den Studien über die Anwendung einer IA im Rahmen von steroidrefraktären Rezidiven zeigte sich eine klinische Besserung in bis zu $85 \%$ der Fälle $[34,51,56,57]$. Insbesondere das Vorhandensein einer Optikus-Neuritis erhöhte das Ansprechen auf eine PP oder IA. Aufgrund der heterogenen Pathophysiologie der MS ist es wünschenswert, die Zugehörigkeit zu den entsprechenden MS-Typen klinisch besser evaluieren zu können. Aktuell bestehen keine diagnostischen Möglichkeiten außerhalb der Biopsie mit anschließender histologischer Untersuchung, um eine Zuordnung zu den pathophysiologischen Typen treffen zu können. Daher ist die Aussagekraft, ob eine IA oder PE bei Patienten mit MS zu einem therapeutischen Erfolg führt, zurzeit begrenzt.

\section{Neuromyelitis optica (NMO)}

Die NMO stellt vermutlich eine autoimmunologisch bedingte seltene inflammatorische Störung des ZNS dar, die hauptsächlich durch eine rezidivierende Optikus-Neuritis und Myelitis auffällt. Aufgrund der Ähnlichkeiten der klinischen Symptome wurde die NMO früher als eine Unterform der MS angesehen. Die NMO muss aber gegen eine MS mit begleitender Optikus-Neuritis abgegrenzt werden $[58,59]$. Von diagnostischer Bedeutung sind Auto-AK gegen Aquaporin-4 (AQP-4), die bei $80 \%$ aller Patienten mit NMO gefunden werden können [60]. Eine PP ist in internationalen neurologischen Leitlinien als eine mögliche therapeutische Alternative bei Therapieversagen der Standardtherapie erwähnt $[43,61,62]$. Einige Fallberichte beschreiben auch den Einsatz der IA [63].

\section{AK-assoziierte limbische Enzephalitis (LE)}

Klinische Symptome einer LE sind episodische Verschlechterungen der Erinnerung, Desorientierung und Agitation verbunden mit epileptischen Anfällen, Halluzinationen, 
Schlafstörungen und dem Nachweis einer temporomedialen Enzephalitis durch radiologische oder histopathologische Untersuchungen [64]. Die LE ist häufig eine paraneoplastische Erkrankung typischerweise im Rahmen von Teratomen [65]. Pathophysiologische Relevanz kommen unterschiedlichen Auto-AK zu. Hierzu zählen Auto-AK gegen den N-Methyl-D-Aspartat-Rezeptor (NMDAR), den spannungsgesteuerten Kaliumkanal (VGKC) oder diverse andere neuronale Antigene [66]. Es können auch autoimmunologisch bedingte Formen ohne eine begleitende Tumorerkrankung vorkommen. Zunehmend können klinische Bilder, die früher als psychiatrisch gewertet wurden, als autoimmunologisch assoziierte neurologische Krankheitsbilder identifiziert werden. Die rechtzeitige Diagnose einer LE mit anschließender immunmodulierender Therapie hat die Prognose der betroffenen Patienten deutlich verbessert [67]. Die therapeutische Apherese hat aufgrund der schnellen und effektiven Elimination der pathogenen Auto-AK einen besonderen Stellenwert innerhalb der Therapie [21] von 30 Patienten, die entweder mit PP oder IA behandelt wurden, zeigten einen therapeutischen Erfolg. Die Anwendung der PP und der IA erscheinen gleichwertig [68].

\section{Akute inflammatorische demyelinisierende Polyradikuloneuropathie (AIDP)}

Die AIDP werden durch autoimmunologische Einflüsse ausgelöst, die über eine akute inflammatorische Reaktion eine Demyelinisierung peripherer motorischer und sensorischer Nervenzellen bewirken. Mit $90 \%$ stellt das Guillain-Barré-Syndrom die häufigste AIDP-Form dar. Es besteht eine Assoziation mit Infektionserregern, wie bspw. Campylobacter jejuni und dem Zika-Virus. Es konnten Auto-AK vom Typ IgG als auch IgM gegen Ganglioside (GM1, GD1a) identifiziert werden, die sowohl durch eine IA als auch eine PP effektiv eliminiert werden können [69]. Die PP stellt dabei eine gleichwertige Alternative zur Gabe von intravenösen Immunglobulinen dar, die sequenzielle Durchführung einer therapeutischen Apherese mit anschließender IVIG-Gabe erbringt keinen zusätzlichen Nutzen. Die IA kann als Alternative zur PP aufgrund einer vergleichbaren Effektivität eingesetzt werden [29]. Nach den aktuellen Cochrane-Daten sollte die therapeutische Apherese innerhalb von 7 Tagen nach Beginn der klinischen Symptome begonnen werden [70].

\section{Chronische inflammatorische demyelini- sierende Polyradikuloneuropathie (CIPD)}

Die CIPD ist eine chronische immunvermittelte inflammatorische neurologische Erkrankung der peripheren Nerven, die entweder rezidivierend oder progressiv verläuft und in mehr als der Hälfte der Fälle zu schweren funktionellen Einbußen führt. Pathophysiologisch spielen Auto-AK gegen Ganglioside oder Proteine des Ranvierschen Schnürrings (u. a. Contactin-1) eine Rolle. Die Mehrzahl der Patienten spricht auf eine immunmodulatorische Therapie an. Primär erscheint die Gabe von IVIG oder die Durchführung
- Tab.7 Auto-Antikörper im Rahmen von lonenkanal-Erkrankungen des peripheren Nervensystems.

\begin{tabular}{|l|l|}
\hline Erkrankung & Auto-Antikörper \\
\hline Myasthenia gravis (MG) & Azetylcholin-Rezeptor \\
\hline & muskelspezifische Tyrosin-Kinase \\
\hline Lambert-Eaton-Myasthenia- & $\begin{array}{l}\text { spannungsgesteuerter Kalziumkanal } \\
\text { (VGCC) }\end{array}$ \\
\hline Syndrom (LEMS) & $\begin{array}{l}\text { spannungsgesteuerter Kaliumkanal } \\
\text { (VGKC) }\end{array}$ \\
\hline
\end{tabular}

eines TPA oder einer IA sinnvoll, die Gabe von Kortison stellt eine therapeutische Alternative dar. Da die CIDP eine heterogene Erkrankung ist, ist auch das Ansprechen auf die unterschiedlichen therapeutischen Ansätze unterschiedlich, wobei bis zu $80 \%$ aller Patienten auf die Primärtherapie respondieren. Nach der Induktionstherapie ist eine Erhaltungstherapie in 40-60\% aller Fälle notwendig, wobei eine kontinuierliche TPA/IA-Behandlung in individuell festzulegenden Abständen eine Alternative darstellt.

\section{Ionen-Kanal-Erkrankungen}

Autoimmunologisch induzierte neurologische lonenkanal-Erkrankungen des peripheren neuromuskulären Systems teilen die pathophysiologische Besonderheit, dass sie durch Auto-AK ausgelöst werden. In $>$ Tab. 7 sind bekannte Auto-AK der jeweiligen Erkrankungen aufgelistet.

\section{Myasthenia gravis (MG)}

Die MG ist eine neurologische Erkrankung, die durch eine gestörte neuromuskuläre Erregungsübertragung an der motorischen Endplatte charakterisiert ist. Klinisches Symptom ist eine Muskelschwäche mit Ermüdbarkeit insbesondere unter zunehmender Belastung. Pathophysiologisch bedeutsam sind Auto-AK gegen den Azetylcholin-Rezeptor (AchR) auf der postsynaptischen Membran, muskelspezifische Tyrosinkinase (MuSK) oder dem LipoproteinRezeptor-related Protein 4 (LRP4) [71-73]. Es besteht eine Assoziation mit dem Vorliegen eines Thymoms. Aufgrund dieser Tatsache ist eine therapeutische Apherese in der akuten und chronischen Therapie sinnvoll und effektiv, insbesondere wenn die bulbäre Muskulatur betroffen ist [72]. Die IA zeigt eine vergleichbare Effektivität zur PP [74, 75]. Obwohl bei einer Vielzahl von Patienten ein Auto-AK nachgewiesen werden kann, stellt auch bei Patienten ohne Nachweis eines Auto-AKs, aber klinischer Diagnose einer MG die Durchführung einer TPA/IA eine Behandlungsoption dar [76].

\section{Fazit}

Mit dem TPA und der IA stehen extrakorporale Therapieverfahren zur Verfügung, mit deren Hilfe pathophysiologisch relevante Mediatoren entfernt werden können. Hier- 
zu zählen insbesondere Auto-AK und AK-Komplexe. Des Weiteren können durch den TPA-Plasmakomponenten in großer Menge zugeführt werden. Neben anderen heutzutage verfügbaren immunmodulierenden Therapien sind der TPA und die IA komplementäre Therapieoptionen. Die Zeit bis zum klinischen Ansprechen auf eine immunmodulierende Therapie kann aufgrund der Halbwertszeit der Zielzellen oder -moleküle mehrere Wochen betragen. Durch den Einsatz eines TPA oder einer IA kann die Zeit bis zur klinischen Besserung verkürzt werden. Größere und vergleichende Studien sind wünschenswert, um die Bedeutung des TPA und der IA bei der Behandlung akuter Erkrankungen mit autoimmunologischer Pathophysiologie zu festigen.

\section{Interessenkonflikt}

Der Autor gibt an, dass kein Interessenkonflikt besteht.

\section{Korrespondenzadresse}

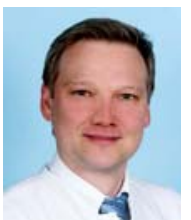

\section{PD Dr. med. Markus Tölle}

CharitéCentrum13 -

Schwerpunkt Nephrologie und Nierentransplantationszentrum

Charité - Universitätsmedizin Berlin

Hindenburgdamm 30

12203 Berlin

markus.toelle@charite.de

Literatur

[1] Stegmayr B, Ramlow W, Balogun RA. Beyond dialysis: current and emerging blood purification techniques. Semin Dial 2012; 25: 207-213. doi:10.1111/j.1525-139X.2011.01034.x

[2] Hirasawa H, Sugai T, Oda S et al. Efficacy and limitation of apheresis therapy in critical care. Ther Apher 1997; 1: 228-232

[3] Huang Z, Wang SR, Su W, Liu JY. Removal of humoral mediators and the effect on the survival of septic patients by hemoperfusion with neutral microporous resin column. Ther Apher Dial 2010; 14: 596-602. doi:10.1111/j.17449987.2010.00825.x

[4] Oishi K, Mimura-Kimura Y, Miyasho T et al. Association between cytokine removal by polymyxin $B$ hemoperfusion and improved pulmonary oxygenation in patients with acute exacerbation of idiopathic pulmonary fibrosis. Cytokine 2013; 61: 84-89. doi:10.1016/j.cyto.2012.08.032

[5] Padmanabhan A, Connelly-Smith L, Aqui N et al. Guidelines on the Use of Therapeutic Apheresis in Clinical Practice - Evidence-Based Approach from the Writing Committee of the American Society for Apheresis: The Eighth Special Issue. J Clin Apher 2019; 34: 171-354. doi:10.1002/jca.21705
[6] Guyatt GH, Oxman AD, Vist GE et al.; GRADE Working Group. GRADE: an emerging consensus on rating quality of evidence and strength of recommendations. BM] 2008; 336: 924-926. doi:10.1136/bmj.39489.470347.AD

[7] Clark WF, Huang SS, Walsh MW et al. Plasmapheresis for the treatment of kidney diseases. Kidney Int 2016; 90: 974-984. doi:10.1016/j.kint.2016.06.009

[8] Bendapudi PK, Hurwitz S, Fry A et al. Derivation and external validation of the PLASMIC score for rapid assessment of adults with thrombotic microangiopathies: a cohort study. Lancet Haematology 2017; 4: e157-e164. doi:10.1016/ S2352-3026(17)30026-1

[9] Tsai HM, Lian EC. Antibodies to von Willebrand factor-cleaving protease in acute thrombotic thrombocytopenic purpura. N Engl J Med 1998; 339: 1585-1594. doi:10.1056/ NEJM199811263392203

[10] Ferrari S, Mudde GC, Rieger M et al. IgG subclass distribution of anti-ADAMTS13 antibodies in patients with acquired thrombotic thrombocytopenic purpura. J Thromb Haemost 2009; 7: 1703-1710. doi:10.1111/j.15387836.2009.03568.x

[11] Coppo P, Schwarzinger M, Buffet M et al. Predictive features of severe acquired ADAMTS13 deficiency in idiopathic thrombotic microangiopathies: the French TMA reference center experience. PloS One 2010; 5: e10208. doi:10.1371/ journal.pone.0010208

[12] Scully M, Hunt B], Benjamin S et al. Guidelines on the diagnosis and management of thrombotic thrombocytopenic purpura and other thrombotic microangiopathies. British J Haematol 2012; 158: 323-335. doi:10.1111/j.13652141.2012.09167.x

[13] Saliba F, Ichaï P, Azoulay D et al. Successful long-term outcome of $\mathrm{ABO}$-incompatible liver transplantation using antigen-specific immunoadsorption columns. Ther Apher Dial 2010; 14: 116-123. doi:10.1111/j.1744-9987.2009.00792.x

[14] Thölking G, Koch R, Pavenstädt $\mathrm{H}$ et al. Antigen-Specific versus Non-Antigen-Specific Immunoadsorption in ABO-Incompatible Renal Transplantation. PLoS One 2015; 10: e0131465. doi:10.1371/journal.pone.0131465

[15] Becker LE, Siebert D, Süsal C et al. Outcomes Following ABO-Incompatible Kidney Transplantation Performed After Desensitization by Nonantigen-Specific Immunoadsorption. Transplantation 2015; 99: 2364-2371. doi:10.1097| TP.0000000000000753

[16] Ahmed T, Senzel L. The role of therapeutic apheresis in the treatment of acute antibody-mediated kidney rejection. J Clin Apher 2012; 27: 173-177. doi:10.1002/jca.21211

[17] Böhmig GA, Wahrmann M, Regele $\mathrm{H}$ et al. Immunoadsorption in severe C4d-positive acute kidney allograft rejection: a randomized controlled trial. Am J Transplant 2007; 7: 117-121. doi:10.1111/j.1600-6143.2006.01613.x

[18] Becker LE, Süsal C, Morath C. Kidney transplantation across $\mathrm{HLA}$ and $\mathrm{ABO}$ antibody barriers. Curr Opin Organ Transplant 2013; 18: 445-454. doi:10.1097/MOT.0b013e3283636c20

[19] Jayne DRW, Gaskin G, Rasmussen N et al.; European Vasculitis Study Group. Randomized Trial of Plasma Exchange or High-Dosage Methylprednisolone as Adjunctive Therapy for Severe Renal Vasculitis. J Am Soc Nephrol 2007; 18: 2180-2188. doi:10.1681/ASN.2007010090

[20] De Lind Van Wijngaarden RA, Hauer HA, Wolterbeek R et al.; EUVAS. Chances of Renal Recovery for Dialysis-Dependent ANCA-Associated Glomerulonephritis. J Am Soc Nephrol 2007; 18: 2189-2197. doi:10.1681/ASN.2007010066 
[21] Palmer A, Cairns T, Dische F et al. Treatment of rapidly progressive glomerulonephritis by extracorporeal immunoadsorption, prednisolone and cyclophosphamide. Nephrol Dial Transplant 1991; 6: 536-542. doi:10.1093/ndt/6.8.536

[22] Stegmayr BG, Almroth G, Berlin G et al. Plasma exchange or immunoadsorption in patients with rapidly progressive crescentic glomerulonephritis. A Swedish multi-center study. Int J Artif Organs 1999; 22: 81-87

[23] Matic G, Michelsen A, Hofmann D et al. Three cases of C-ANCA-positive vasculitis treated with immunoadsorption: possible benefit in early treatment. Ther Apher 2001; 5: 68-72

[24] Fencl F, Simková E, Vondrák K et al. Recurrence of nephrotic proteinuria in children with focal segmental glomerulosclerosis after renal transplantation treated with plasmapheresis and immunoadsorption: case reports. Transplantation Proc 2007; 39: 3488-3490. doi:10.1016/j. transproceed.2007.09.045

[25] Morath C, Wei C, Macher-Goeppinger S et al. Management of severe recurrent focal segmental glomerulosclerosis through circulating soluble urokinase receptor modification. Am J Ther 2013; 20: 226-229. doi:10.1097| MJT.0b013e3182811aca

[26] Lionaki S, Vlachopanos G, Georgalis A et al. Individualized scheme of immunoadsorption for the recurrence of idiopathic focal segmental glomerulosclerosis in the graft: a single center experience. Ren Fail 2015; 37: 777-783. doi:10.3109/ 0886022X.2015.1015366

[27] Beaudreuil S, Zhang X, Kriaa F et al. Protein A immunoadsorption cannot significantly remove the soluble receptor of urokinase from sera of patients with recurrent focal segmental glomerulosclerosis. Nephrol Dial Transplant 2014; 29: 458-463. doi:10.1093/ndt/gft453

[28] Köhler W, Bucka C, Klingel R. A randomized and controlled study comparing immunoadsorption and plasma exchange in myasthenic crisis. J Clin Apher 2011; 26: 347-355. doi:10.1002/jca.20317

[29] Galldiks N, Dohmen C, Neveling M et al. Selective immune adsorption treatment of severe Guillain Barre syndrome in the intensive care unit. Neurocrit Care 2009; 11: 317-321. doi:10.1007/s12028-009-9252-6

[30] Seta T, Nagayama H, Katsura K et al. Factors influencing outcome in Guillain-Barre Syndrome: comparison of plasma adsorption against other treatments. Clin Neurol Neurosurg 2005; 107: 491-496. doi:10.1016/j.clineuro.2004.12.019

[31] Bramlage CP, Schröder K, Bramlage P et al. Predictors of complications in therapeutic plasma exchange. J Clin Apher 2009; 24: 225-231. doi:10.1002/jca.20217

[32] Belàk M, Borberg H, Jimenez C, Oette K. Technical and clinical experience with protein A immunoadsorption columns. Transfus Sci 1994; 15: 419-422. doi:10.1016/09553886(94)90174-0

[33] Yeh JH, Chiu HC. Comparison between double-filtration plasmapheresis and immunoadsorption plasmapheresis in the treatment of patients with myasthenia gravis. J Neurol 2000; 247: 510-513. doi:10.1007/s004150070149

[34] Koziolek M], Tampe D, Bähr M et al. Immunoadsorption therapy in patients with multiple sclerosis with steroid-refractory optical neuritis. J Neuroinflammation 2012; 9: 80. doi:10.1186/1742-2094-9-80

[35] Garg RK. Acute disseminated encephalomyelitis. Postgrad Med J 2003; 79: 11-17. doi:10.1136/pmj.79.927.11

[36] Kaynar L, Altuntas F, Aydogdu I et al. Therapeutic plasma exchange in patients with neurologic diseases: retrospective multicenter study. Transfus Apher Sci 2008; 38: 109-115. doi:10.1016/j.transci.2007.11.002

[37] Keegan M, Pineda AA, McClelland RL et al. Plasma exchange for severe attacks of CNS demyelination: Predictors of response. 2002; 58: 143-146. doi:10.1212/wnl.58.1.143

[38] Noseworthy JH, Lucchinetti C, Rodriguez M, Weinshenker BG. Multiple sclerosis. N Engl J Med 2000; 343: 938-952. doi:10.1056/NEJM200009283431307

[39] Szczepiorkowski ZM, Winters JL, Bandarenko N et al.; Apheresis Applications Committee of the American Society for Apheresis. Guidelines on the use of therapeutic apheresis in clinical practice--evidence-based approach from the Apheresis Applications Committee of the American Society for Apheresis. J Clin Apher 2010; 25: 83-177. doi:10.1002/ jca.20240

[40] Wilson HL. B cells contribute to MS pathogenesis through antibody-dependent and antibody-independent mechanisms. Biologics 2012; 6: 117-123. doi:10.2147/BTT.S24734

[41] Keegan M, König F, McClelland R et al. Relation between humoral pathological changes in multiple sclerosis and response to therapeutic plasma exchange. Lancet 2005; 366 : 579-582. doi:10.1016/50140-6736(05)67102-4

[42] Meuth SG, Göbel K, Wiendl H. Immune therapy of multiple sclerosis--future strategies. Curr Pharm Des 2012; 18: 4489-4497. doi:10.2174/138161212802502198

[43] Multiple Sclerosis Therapy Consensus Group (MSTCG), Wiendl H, Toyka KV, Rieckmann P et al. Basic and escalating immunomodulatory treatments in multiple sclerosis: current therapeutic recommendations. J Neurol 2008; 255: 1449-1463. doi:10.1007/s00415-008-0061-1

[44] Reindl M, Linington C, Brehm U et al. Antibodies against the myelin oligodendrocyte glycoprotein and the myelin basic protein in multiple sclerosis and other neurological diseases: a comparative study. Brain 1999; 122: 2047-2056. doi:10.1093/brain/122.11.2047

[45] Berger T. Is there a rationale for therapeutic immunoadsorption in multiple sclerosis? Eur J Clin Invest 2005; 35: 467-468. doi:10.1111/j.1365-2362.2005.01546.x

[46] Kuerten S, Pauly R, Blaschke $S$ et al. [The significance of a B cell-dependent immunopathology in multiple sclerosis]. Fortschr Neurol Psychiatr 2011; 79: 83-91. doi:10.1055/s-0029-1245937

[47] Klingel R, Heibges A, Fassbender C. Plasma exchange and immunoadsorption for autoimmune neurologic diseases - current guidelines and future perspectives. Atheroscler Suppl 2009; 10: 129-132. doi:10.1016/S1567-5688(09)71827-6

[48] Weiner HL, Dau PC, Khatri BO et al. Double-blind study of true vs. sham plasma exchange in patients treated with immunosuppression for acute attacks of multiple sclerosis. Neurology 1989; 39: 1143-1149. doi:10.1212/ wnl.39.9.1143

[49] Weinshenker BG, O’Brien PC, Petterson TM et al. A randomized trial of plasma exchange in acute central nervous system inflammatory demyelinating disease. Ann Neurol 1999; 46: 878-886

[50] Magaña SM1, Keegan BM, Weinshenker BG et al. Beneficial plasma exchange response in central nervous system inflammatory demyelination. Arch Neurol 2011; 68: 870-878. doi:10.1001/archneurol.2011.34

[51] Trebst C, Bronzlik P, Kielstein JT et al. Immunoadsorption therapy for steroid-unresponsive relapses in patients with multiple sclerosis. Blood Purif 2012; 33: 1-6. doi: $10.1159 / 000332397$ 
[52] Linker RA, Chan A, Sommer M et al. Plasma exchange therapy for steroid-refractory superimposed relapses in secondary progressive multiple sclerosis. J Neurol 2007; 254: 1288-1289. doi:10.1007/s00415-006-0497-0

[53] Gold R. Diagnose und Therapie der Multiplen Sklerose. In: Diener HC, P. N., Hrsg. Leitlinien für Diagnostik und Therapie in der Neurologie. 5. Aufl. 2012, letztes Online-Update: 2014

[54] Schröder A, Fischer M, Meyer C et al. Therapeutic plasma exchange for steroid unresponsive multiple sclerosis relapss - a long-term follow-up observation including 35 patients. Akt Neurol 2009; 36: 105-110. doi:10.1055/s-0028-1090231

[55] Trebst C, Reising A, Kielstein JT et al. Plasma exchange therapy in steroid-unresponsive relapses in patients with multiple sclerosis. Blood Purif 2009; 28: 108-115. doi:10.1159/000224630

[56] de Andrés C, Anaya F, Giménez-Roldan S. [Plasma immunoadsorption treatment of malignant multiple sclerosis with severe and prolonged relapses]. Rev Neurol 2000; 30 : 601-605

[57] Mauch E, et al. [Immunoadsorption for steroid-unresponsive multiple sclerosis-relapses: clinical data of 14 patients]. Nervenarzt 2011; 82: 1590-1595. doi:10.1007/s00115-0113324-5

[58] Jarius S, Ruprecht K, Wildemann B et al. Contrasting disease patterns in seropositive and seronegative neuromyelitis optica: A multicentre study of 175 patients. J Neuroinflammation 2012; 9: 14. doi:10.1186/1742-2094-9-14

[59] Trebst C, Berthele A, Jarius S et al. [Diagnosis and treatment of neuromyelitis optica. Consensus recommendations of the Neuromyelitis Optica Study Group]. Nervenarzt 2011; 82: 768-777. doi:10.1007/s00115-010-3192-4

[60] Jarius S, Wildemann B, Paul F. Neuromyelitis optica: clinical features, immunopathogenesis and treatment. Clin Exp Immunol 2014; 176: 149-164. doi:10.1111/cei.12271

[61] Cortese I, Chaudhry V, So YT et al. Evidence-based guideline update: Plasmapheresis in neurologic disorders: report of the Therapeutics and Technology Assessment Subcommittee of the American Academy of Neurology. Neurology 2011; 76: 294-300. doi:10.1212/WNL.0b013e318207b1f6

[62] Cortese I, Cornblath DR. Therapeutic plasma exchange in neurology: 2012.J Clin Apher 2013; 28: 16-19. doi:10.1002| jca. 21266

[63] Hoffmann F, Kraft A, Heigl F et al. [Tryptophan immunoadsorption for multiple sclerosis and neuromyelitis optica: therapy option for acute relapses during pregnancy and breastfeeding]. Nervenarzt 2015; 86: 179-186. doi:10.1007| s00115-014-4239-8

[64] Vincent A, Buckley C, Schott JM et al. Potassium channel antibody-associated encephalopathy: a potentially immunotherapy-responsive form of limbic encephalitis. Brain 2004; 127: 701-712. doi:10.1093/brain/awh077

[65] Graus F, Saiz A, Lai M et al. Neuronal surface antigen antibodies in limbic encephalitis: clinical-immunologic associations. Neurology 2008; 71: 930-936. doi:10.1212/01. wnl.0000325917.48466.55

[66] Anderson NE, Barber PA. Limbic encephalitis - a review. J Clin Neurosci 2008; 15: 961-971. doi:10.1016/j. jocn.2007.12.003

[67] Friese MA, Magnus T. [Autoimmune synaptic encephalopathies]. Nervenarzt 2011; 82: 1239-1249. doi:10.1007| s00115-010-3219-x

[68] Ehrlich S, Fassbender CM, Blaes C et al. [Therapeutic apheresis for autoimmune encephalitis: a nationwide data collection]. Nervenarzt 2013; 84: 498-507. doi:10.1007| s00115-012-3710-7

[69] Marn Pernat A, Buturovic-Ponikvar ], Svigelj V, Ponikvar R. Guillain-Barré syndrome treated by membrane plasma exchange and/or immunoadsorption. Ther Apher Dial 2009; 13 : 310-313. doi:10.1111/j.1744-9987.2009.00730.x

[70] Chevret S, Hughes RA, Annane D. Plasma exchange for Guillain-Barré syndrome. Cochrane Database Syst Rev 2017; 2: CD001798. doi:10.1002/14651858.CD001798.pub3

[71] Meriggioli MN, Sanders DB. Autoimmune myasthenia gravis: emerging clinical and biological heterogeneity. Lancet Neurol 2009; 8: 475-490. doi:10.1016/S1474-4422(09)70063-8

[72] Antozzi C, Gemma M, Regi B et al. A short plasma exchange protocol is effective in severe myasthenia gravis. J Neurol 1991; 238: 103-107. doi:10.1007/bf00315690

[73] Guptill JT, Sanders DB, Evoli A. Anti-MuSK antibody myasthenia gravis: clinical findings and response to treatment in two large cohorts. Muscle Nerve 2011; 44: 36-40. doi:10.1002/ mus.22006

[74] Antozzi C, Berta E, Confalonieri P et al. Protein-A immunoadsorption in immunosuppression-resistant myasthenia gravis. Lancet 1994; 343: 124

[75] Grob D, Simpson D, Mitsumoto $\mathrm{H}$ et al. Treatment of myasthenia gravis by immunoadsorption of plasma. Neurology 1995; 45: 338-344. doi:10.1212/wnl.45.2.338

[76] Leite MI, Jacob S, Viegas S et al. IgG1 antibodies to acetylcholine receptors in 'seronegative' myasthenia gravis. Brain 2008; 131: 1940-1952. doi:10.1093/brain/awn092 